\title{
Correction: The nuclear hypoxia-regulated NLUCAT1 long non- coding RNA contributes to an aggressive phenotype in lung adenocarcinoma through regulation of oxidative stress
}

\author{
Laura Moreno Leon - Marine Gautier · Richard Allan - Marius Ilié $(\mathbb{D} \cdot$ Nicolas Nottet • Nicolas Pons · Agnes Paquet • \\ Kévin Lebrigand · Marin Truchi · Julien Fassy • Virginie Magnone · Garrett Kinnebrew • Milan Radovich •

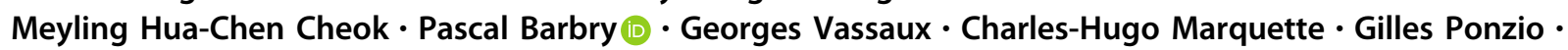 \\ Mircea Ivan • Nicolas Pottier • Paul Hofman • Bernard Mari (i) Roger Rezzonico
}

Published online: 8 March 2021

(c) The Author(s), under exclusive licence to Springer Nature Limited 2021

Correction to: Oncogene
https://doi.org/10.1038/s41388-019-0935-y

The original version of this article unfortunately contained a mistake. The following correction has therefore been made in the original: The term "LUCAT1" was missing in the abstract. The corrected abstract is given below. The original article has been corrected.

\section{Abstract}

Lung cancer is the leading cause of cancer death worldwide, with poor prognosis and a high rate of recurrence despite early surgical removal. Hypoxic regions within tumors represent sources of aggressiveness and resistance to therapy. Although long non-coding RNAs (lncRNAs) are increasingly recognized as major gene expression regulators, their regulation and function following hypoxic stress are still largely unexplored. Combining profiling studies on early-stage lung adenocarcinoma (LUAD) biopsies and on A549 LUAD cell lines cultured in normoxic or hypoxic conditions, we identified a subset of lncRNAs that are both correlated with the hypoxic status of tumors and regulated by hypoxia in vitro. We focused on a new transcript, Nuclear LUCAT1 (NLUCAT1), which is strongly upregulated by hypoxia in vitro and correlated with hypoxic markers and poor prognosis in LUADs. Full molecular characterization showed that NLUCAT1 is a large nuclear transcript composed of six exons and mainly regulated by NF- $\mathrm{KB}$ and NRF2 transcription factors. CRISPR-Cas9-mediated invalidation of NLUCAT1 revealed a decrease in proliferative and invasive properties, an increase in oxidative stress and a higher sensitivity to cisplatin-induced apoptosis. Transcriptome analysis of NLUCAT1-deficient cells showed repressed genes within the antioxidant and/or cisplatin-response networks. We demonstrated that the concomitant knockdown of four of these genes products, GPX2, GLRX, ALDH3A1, and PDK4, significantly increased ROS-dependent caspase activation, thus partially mimicking the consequences of NLUCAT1 inactivation in LUAD cells. Overall, we demonstrate that NLUCAT1 contributes to an aggressive phenotype in early-stage hypoxic tumors, suggesting it may represent a new potential therapeutic target in LUADs. 